\title{
Time-dependent Simulations of Quantum Waveguides Using a Time-Splitting Spectral Method ${ }^{\text {出 }}$
}

\author{
Ansgar Jüngel $^{1}$, Jan-Frederik Mennemann ${ }^{1}$ \\ ${ }^{a}$ Institute for Analysis and Scientific Computing, Vienna University of Technology, Wiedner Hauptstraße \\ 8-10, 1040 Wien, Austria
}

\begin{abstract}
The electron flow through quantum waveguides is modeled by the time-dependent Schrödinger equation with absorbing boundary conditions, which are realized by a negative imaginary potential. The Schrödinger equation is discretized by a time-splitting spectral method, and the quantum waveguides are fed by a mono-energetic incoming plane wave pulse. The resulting algorithm is extremely efficient due to the Fast Fourier Transform implementation of the spectral scheme. Numerical convergence rates for a one-dimensional scattering problem are calculated. The transmission rates of a two-dimensional T-stub quantum waveguide and a single-branch coupler are numerically computed. Moreover, the transient behavior of a three-dimensional T-stub waveguide is simulated.
\end{abstract}

Keywords: Spectral method, Schrödinger equation, absorbing boundary conditions, quantum waveguide, quantum transistor, three-dimensional dynamical simulations. 2000 MSC: : 65M70, 35Q40, 37N20.

\section{Introduction}

Nanoscale quantum electronics is a promising research field for devising new device classes with a high degree of functionality. The electron transport in these devices can be considered to be ballistic at low temperatures. Then the electrical properties depend on quantum interferences, controlled by the bias voltage applied to the gate contacts. These devices may be used as nanoscale electronic switches, quantum interference transistors, multiplexers etc. $[10,35,39]$. They are made of different semiconductor materials in such a way that the electrons are confined to small channels or waveguides. Due to the strong confinement of the electron gas to one or two space dimensions, the ballistic quantum

\footnotetext{
The authors acknowledge partial support from the Austrian Science Fund (FWF), grant P20214 and WK "Differential Equations", the German Science Foundation (DFG), grant JU 359/7, the Austria-Croatia Project HR 01/2010, the Austria-France Project FR 07/2010, and the Austria-Spain Project ES 08/2010 of the Austrian Exchange Service (ÖAD).

Email addresses: juengel@anum.tuwien.ac.at (Ansgar Jüngel), mennemann@anum.tuwien.ac.at (Jan-Frederik Mennemann)
} 
transport may be modeled by the one-dimensional or two-dimensional Schrödinger equation. Since there are quantum effects which can be only explained in a three-dimensional model [37], also three-dimensional simulations are of interest.

In this paper, the electron transport in quantum waveguides is modeled by the timedependent Schrödinger equation with open boundary conditions, which is suitable for ballistic transport. An alternative approach consists in the solution of the nonequilibrium Green's function equations (coupled selfconsistently with the Poisson equation), see, e.g., $[36,40]$. Although the open boundary Schrödinger and non-equilibrium Green's functions approaches can be considered to be formally equivalent, their numerical treatment is different [30]. The dynamical behavior of the electrons in a quantum waveguide has been also described by Wigner function models [38]. The disadvantage of Wigner models is its high dimensionality, requiring to solve a six-dimensional problem in three-dimensional devices.

We discretize the Schrödinger equation by employing a time-splitting spectral method. More precisely, the Schrödinger equation is split into the free Schrödinger equation and an ordinary differential equation for the electric potential (see Section 2). The latter equation can be solved explicitly, whereas the former one is approximated by a spectral method $[8,27]$. There are several time-splitting strategies such as the Trotter and Strang splitting; see [17] for the corresponding error estimates. These methods are unconditionally stable, mass conservative, and gauge invariant [8]. Another advantage of the spectral method is that the discrete set of equations can be solved very efficiently by using the Fast Fourier Transform. In fact, the complexity of the full algorithm for one time step is of the order $\mathcal{O}(M \log M)$, where $M=M_{1} \cdots M_{d}$ and $M_{j}$ is the number of grid points in the $j$-th direction (see Section 2).

For the open boundary problem it is necessary to devise appropriate absorbing boundary conditions at the interface between the leads and the active device domain in order to avoid unphysical reflections at the boundary. In the literature, there are several ways of deriving absorbing or transparent boundary conditions (TBC). Analytic TBC are nonlocal in time, and their numerical implementation requires some care (see the review [1] and references therein). Moreover, inadequate discretizations may introduce strong reflections at the boundary [12].

In the quantum transmitting boundary method, the contact region along the leads is replaced by outgoing waveguide modes with known transmitting characteristics [20, 30]. This method needs to sum up a large number of modes of the incident, reflected, and transmitted waves from each terminal, and therefore, its implementation is rather complex.

Another method is to introduce perfectly matched layers, first developed for the Maxwell equations [9]. The idea is to enlarge the computational domain by an artificial damping layer of finite width, where a modified equation has to be solved. The method has been applied to the Schrödinger equation in $[11,26]$.

A different idea has been presented in [34]. The (periodic) wave function is decomposed periodically in time into a family of coherent states. In contrast to perfectly matched layer methods, the phase-space filter method of [34] filters only those regions of the phase space containing outgoing waves. However, like for perfectly matched layer methods, it is not easy to treat waves with low kinetic energy. 
In the context of finite-difference discretizations such as the Crank-Nicolson method, discrete TBC have been derived [3]. They yield unconditionally stable numerical schemes which are completely reflection-free at the boundary [6]. Discrete TBC include the discrete convolution of the unknown function with a given kernel, and hence, its numerical computation is rather involved. The evaluation of discrete TBC can be significantly accelerated by approximating the kernel by a finite sum of exponentials that decay with respect to time [6]. The limit of vanishing spatial approximation parameters in the discrete TBC coincides with the temporally semi-discrete TBC of Schmidt and Deuflhard [32] and of Lubich and Schädle [21, 22, 31]. For more references on TBC, we refer to [1, 41].

Our aim is to develop a fast, easy-to-implement numerical scheme for the open Schrödinger problem in up to three space dimensions. Therefore, in order to reduce the computational cost, we have chosen to model absorbing boundary conditions by the simple imaginary absorbing potential method. Negative imaginary potentials have been proposed to damp the wave function before it reaches the boundary of the computational domain $[14,19,23,25]$. In [25] criteria for selecting the optimal height and width of such potentials are investigated. It turns out that for low-energy waves, the computational domain has to be large compared to the physical domain, thus increasing the computational cost. However, due to the low complexity of the time-splitting spectral scheme, the overall complexity is still low, and three-dimensional simulations of quantum transistors are possible on modern standard PCs within a few hours of computing time (see Section 3).

Surprisingly, there are only few results in the literature on dynamical simulations of quantum devices in two and three space dimensions. In fact, most of the numerical results (in three dimensions) concern the stationary Schrödinger equation, see e.g. [11, 30, 33]. Transient simulations are typically performed in one or two space dimensions only, see e.g. [7, 34] for Schrödinger simulations and [24] for Wigner simulations. In [15], the timedependent Schrödinger equation is numerically solved in three space dimensions, but no devices have been simulated. In this paper, we present dynamical simulations of a threedimensional quantum device.

Our work is organized as follows. In Section 2, we detail the time-splitting spectral method, the choice of the negative imaginary potential, and the injection of a continuously incoming mono-energetic plane wave pulse. Section 3 is devoted to the numerical examples. First, we perform a numerical test in one space dimension to compute numerical convergence rates. Furthermore, in two space dimensions, the stationary behavior in a T-stub interference transistor and a single-branch coupler are simulated. The numerical results are compared with those from [10]. Moreover, the dynamics of a three-dimensional T-stub waveguide are presented.

\section{Numerical scheme}

We consider the time-dependent Schrödinger equation for the wave function $u$ :

$$
i \hbar \partial_{t} u=-\frac{\hbar^{2}}{2 m^{*}} \Delta u+V(x) u, \quad \text { in } \Omega, t>0, \quad u(\cdot, 0)=u_{0} \quad \text { in } \Omega
$$


where $\Omega \subset \mathbb{R}^{d}(d \leq 3)$ is a bounded domain. The physical constants are the reduced Planck constant $\hbar$ and the effective mass $m^{*}$, and $V$ denotes the external potential. The device domain is generally included in the computational domain $\Omega$ which is taken as a rectangle or cuboid in the simulations. We prescribe periodic boundary conditions on $\Omega$.

In this paper, the potential is a given function and models the confinement structure (as in [7]). It is possible to couple the Schrödinger equation selfconsistently with the Poisson equation; see, e.g. [29, 30].

\subsection{Numerical discretization}

Let $\Omega=\Pi_{j=1}^{3}\left(a_{j}, b_{j}\right)$ be a cuboid. We introduce the spatial grid $\left(x_{\alpha}\right)=\left(x_{1}^{\left(\alpha_{1}\right)}, x_{2}^{\left(\alpha_{2}\right)}\right.$, $\left.x_{3}^{\left(\alpha_{3}\right)}\right)$, where $x_{j}^{\left(\alpha_{j}\right)}=a_{j}+\alpha_{j} \triangle x_{j}, \triangle x_{j}=\left(b_{j}-a_{j}\right) / M_{j}, \alpha_{j} \in\left\{0, \ldots, M_{j}-1\right\}$, and $M_{j}$ are even integers $(j=1,2,3)$. The time steps are given by $t_{n}=n \Delta t$, where $\Delta t>0$ and $n \in \mathbb{N}$. We denote by $u_{\alpha}^{n}$ the numerical approximation of $u\left(x_{\alpha}, t_{n}\right)$. In this notation, $\alpha=\left(\alpha_{1}, \alpha_{2}, \alpha_{3}\right)$ denotes a multiindex with $\alpha \in I:=\Pi_{j=1}^{3}\left\{0, \ldots, M_{j}-1\right\}$.

The solution to the Schrödinger equation (1) at time $t_{n+1}$ with initial datum $u\left(t_{n}\right)$ can be formally written as $u\left(t_{n+1}\right)=e^{-i(A+B) \Delta t} u\left(t_{n}\right)$, where $A=-\left(\hbar / 2 m^{*}\right) \Delta$ and $B=V(x) / \hbar$, and $u(t)$ denotes the function $u(\cdot, t)$. The Trotter splitting is based on the approximation

$$
u\left(t_{n+1}\right) \approx e^{-i B \triangle t} e^{-i A \triangle t} u\left(t_{n}\right),
$$

i.e., first the free Schrödinger equation is solved for one time step,

$$
i \hbar \partial_{t} u_{1}=-\frac{\hbar^{2}}{2 m^{*}} \Delta u_{1}, \quad u_{1}(0)=u\left(t_{n}\right), \quad t>0
$$

followed by solving the ordinary differential equation

$$
i \hbar \partial_{t} u_{2}=V(x) u_{2}, \quad u_{2}(0)=u_{1}(\triangle t), \quad t>0,
$$

for another time step. Then $u_{2}(\triangle t)$ is an approximation of $u\left(t_{n+1}\right)$.

An alternative approximation is the Strang splitting

$$
u\left(t_{n+1}\right) \approx e^{-i B \triangle t / 2} e^{-i A \triangle t} e^{-i B \triangle t / 2} u\left(t_{n}\right) .
$$

Convergence of this approximation follows from the Trotter product formula, and error estimates have been derived in $[8,17]$. Equation (2) will be discretized in space by the spectral method and integrated in time exactly. Then (3) is solved exactly. The method is given as follows. Let the Fourier coefficients of $u_{\alpha}^{n}$ be given by

$$
\widehat{u}_{k}^{n}=\sum_{\alpha \in I} u_{\alpha}^{n} e^{-i \mu_{k} \cdot\left(x_{\alpha}-a\right)}, \quad \mu_{k}=\left(\mu_{k, 1}, \mu_{k, 2}, \mu_{k, 3}\right), \quad \mu_{k, j}=\frac{2 \pi k_{j}}{b_{j}-a_{j}},
$$

where $k=\left(k_{1}, k_{2}, k_{3}\right) \in J:=\prod_{j=1}^{3}\left\{-M_{j} / 2, \ldots, M_{j} / 2-1\right\}$ and $a=\left(a_{1}, a_{2}, a_{3}\right)$. Then, given $\widehat{u}_{k}^{n}$ at time step $t_{n}$, the approximation at step $t_{n+1}$ is computed from

$$
\widehat{u}_{\alpha}^{n+1}=\frac{1}{M} e^{-i V\left(x_{\alpha}\right) \Delta t / \hbar} \sum_{k \in J} e^{-i \hbar\left|\mu_{k}\right|^{2} \Delta t / 2 m^{*}} \widehat{u}_{k}^{n} e^{i \mu_{k} \cdot\left(x_{\alpha}-a\right)},
$$


where $M=M_{1} M_{2} M_{3}$. The initial value is given by

$$
u_{\alpha}^{0}=u\left(x_{\alpha}, 0\right)=u_{0}\left(x_{\alpha}\right), \quad \alpha \in I .
$$

The Strang time splitting is similar to the Trotter splitting. Indeed, $n$ steps of the Strang splitting can be reformulated as $n$ steps of the Trotter splitting framed by $e^{-i B \triangle t / 2}$, i.e.

$$
u_{n}^{\text {Strang }}=e^{-i B \triangle t / 2}\left(e^{-i B \Delta t} e^{-i A \Delta t}\right)^{n} e^{-i B \Delta t / 2} u\left(t_{0}\right) .
$$

The symmetric structure of the Strang splitting increases the order from 1 (Trotter) splitting to 2 (Strang). As the computational work is nearly the same, the Strang splitting is clearly preferable (see Section 3.1).

In the simulations below, the above algorithm is implemented in the Matlab programming language. The discrete Fourier transforms are computed by the Fast Fourier Transform (FFT) based on the FFTW library [13]. This FFT implementation is fastest when the length of the vector is a power of two, but it is almost as fast for lengths which have small prime factors. Thus, the numbers $M_{1}, M_{2}$, and $M_{3}$ do not necessarily need to be powers of two. This fact is employed particularly in our two- and three-dimensional simulations. The complexity of the time-splitting spectral method for one time step is dominated by the $d$-dimensional FFT and is therefore of the order $\mathcal{O}(M \log M)$.

\subsection{Absorbing boundary conditions}

Due to the periodic boundary conditions, wave packets will, after some time, wrap around the computational domain which is not physical for our applications. One way to overcome this problem is to damp the wave function before it reaches the boundary of the computational domain by using a complex potential with negative imaginary part in the vicinity of the boundaries. Other methods for designing absorbing boundary conditions have been described in the introduction. Our choice is based on the observation that the resulting numerical scheme can be solved very efficiently.

Negative imaginary potentials have been employed in quantum chemistry computations since several years; see, e.g. [19] and the references in [16, 23]. In [25], a linear imaginary potential has been suggested, and criteria for selecting the optimal height and width of the potential have been derived. The Saxon-Woods potential $V_{\text {abs }}(x)=-i V_{0} /\left(1+e^{\alpha(x-a)}\right)$ has been used in [19] for studies of the multiphonon dissiciation in diatomic molecules. Since it is generally difficult to absorb outgoing waves with low frequency, some authors have added a negative real potential to increase the kinetic energy of the wave function, thereby reducing its de Broglie wave length. For instance, a complex absorbing potential with power functions has been chosen in [14], whereas in [16] exponential functions have been taken.

In three dimensions, assuming that the wave packet travels in $x_{1}$-direction, we use a 
potential of quadratic type

$$
V_{\mathrm{abs}}\left(x_{1}, x_{2}, x_{3}\right)= \begin{cases}-i V_{0}\left(\frac{x_{1}-x_{1}^{\ell}}{a_{1}-x_{1}^{\ell}}\right)^{2} & \text { if } x_{1}<x_{1}^{\ell}, \\ 0 & \text { if } x_{1}^{\ell} \leq x_{1}<x_{1}^{r}, \\ -i V_{0}\left(\frac{x_{1}-x_{1}^{r}}{b_{1}-x_{1}^{r}}\right)^{2} & \text { if } x_{1}^{r} \leq x_{1},\end{cases}
$$

where $x_{1}^{\ell}$ and $x_{1}^{r}\left(x_{1}^{\ell}<x_{1}^{r}\right)$ denote the boundaries of the device domain in the $x_{1}$-direction. As an example, the imaginary part of (4) is presented in Figure 1. In all of the following simulations, we have chosen $V_{0}=50 \mathrm{meV}, a_{1}=-100 \mathrm{~nm}, b_{1}=180 \mathrm{~nm}, x_{1}^{\ell}=0 \mathrm{~nm}$, and $x_{1}^{r}=80 \mathrm{~nm}$. Thus, in the simulations, we replace the external potential $V$ in the Schrödinger equation (1) by $V+V_{\text {abs }}$.

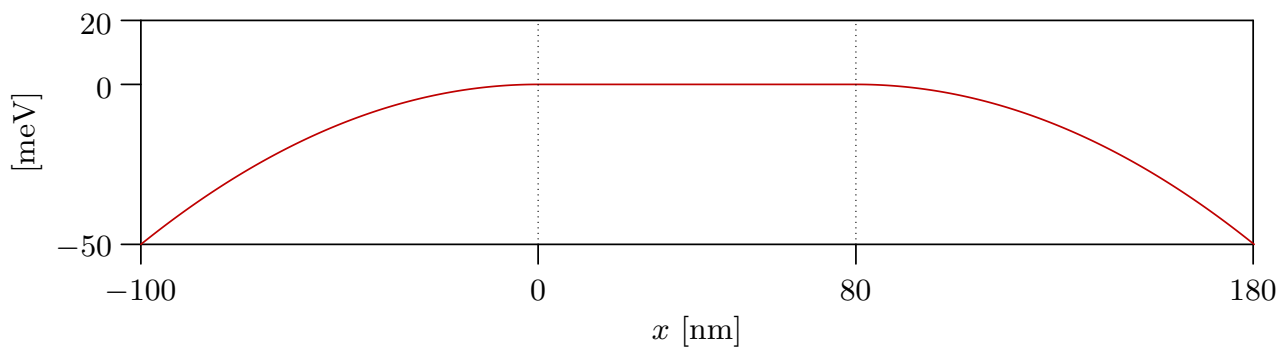

Figure 1: Imaginary part of the absorbing potential (4) with $V_{0}=50 \mathrm{meV}$.

As a numerical example, the evolution of a free Gaussian wave packet in one space dimension under the influence of the potential (4) is illustrated in Figure 2. The wave packet (dashed line) is given by

$$
u(x, t)=\left(1+i \frac{t}{\tau}\right)^{-1 / 2} \exp \left[\left(1+i \frac{t}{\tau}\right)^{-1}\left(-\left(\frac{x-x_{s}}{2 \sigma}\right)^{2}+i k\left(x-x_{s}\right)-i \sigma^{2} k^{2} \frac{t}{\tau}\right)\right],
$$

where $\tau=2 m^{*} \sigma^{2} / \hbar, k=\sqrt{2 m^{*} E} / \hbar, x_{s}=40 \mathrm{~nm}, \sigma=10 \mathrm{~nm}$, and $E=16.74 \mathrm{meV}$.

The effective mass is $m^{*}=0.067 m_{0}$, where $m_{0}$ is the electron mass at rest, corresponding to GaAs. The other wave packet in Figure 2 (solid line) is computed from the Strang time-splitting spectral scheme, with $\triangle x=0.25 \mathrm{~nm}$ and $\triangle t=0.25 \mathrm{fs}$, using the complex absorbing potential (4). The device domain is the interval $[0,80 \mathrm{~nm}]$, whereas the computational domain extends from $-100 \mathrm{~nm}$ to $180 \mathrm{~nm}$ and is more than three times larger than the physical domain. This ensures that the numerical solution (solid line) is indistinguishable from the exact solution (dashed line) in the physical domain.

The drawbacks of absorbing potentials are that they require an enlarged computational domain to absorb the wave function and that they have to be fitted to the frequency range of the incoming wave packets. However, since the numerical algorithm is dominated by the $\mathcal{O}(M \log M)$ complexity of the time-splitting spectral scheme, the additional computational effort due to the enlarged domain is less important. Moreover, the algorithm is not difficult to implement and can be parallelized easily. In order to met the second drawback, we 

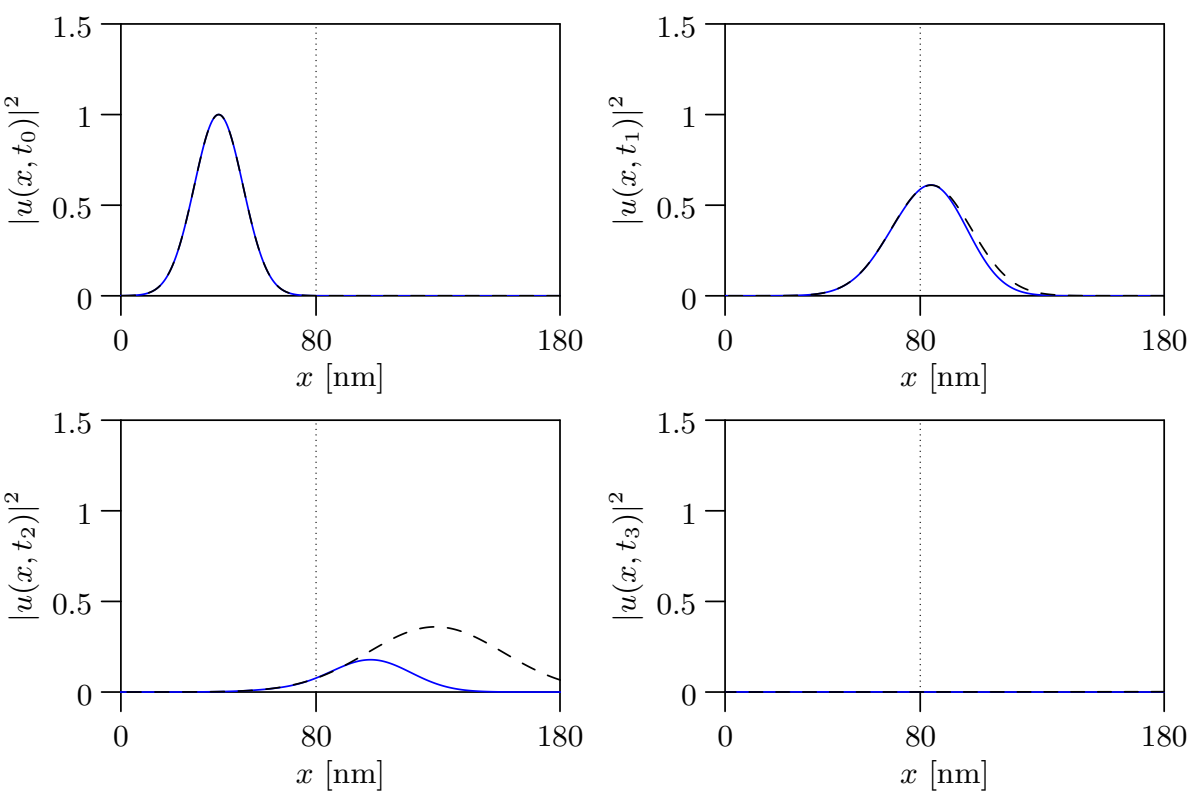

Figure 2: Propagation of a Gaussian wave packet under the influence of the potential (4) with averaged kinetic energy $16.74 \mathrm{meV}$ at times $0,150 \mathrm{fs}$ (upper row) and $300 \mathrm{fs}, 2 \mathrm{ps}$ (lower row). Solid line: numerical solution, dashed line: exact solution.

utilize large absorbing regions outside the quantum device, in which the absorbing potential increases smoothly. This allows us to absorb wave packets over a broad spectrum of momenta and for low kinetic energies (see Section 3 for details).

\subsection{Injection of plane waves}

The incoming electrons are modeled by the mono-energetic plane waves $e^{i(k \cdot x-\omega t)}$, which describe electrons with kinetic energy $E=\hbar^{2}|k|^{2} / 2 m^{*}$ and angular frequency $\omega=E / \hbar$. More precisely, we create a continuously incoming plane wave pulse spreading from the incoming lead contact to the outgoing leads. In one space dimension, we start with the wave function

$$
u_{j}^{0}= \begin{cases}\phi\left(x_{j}\right) & \text { if } x_{j}<0, \\ e^{i k x_{j}}\left(\frac{1}{2}+\frac{1}{2} \cos \left(x_{j} \pi / \delta\right)\right) & \text { if } 0 \leq x_{j}<\delta \\ 0 & \text { if } \delta \leq x_{j} .\end{cases}
$$

Here, $\phi$ denotes the numerical solution of the stationary Schrödinger equation

$$
-\frac{\hbar^{2}}{2 m^{\star}} \frac{\partial^{2}}{\partial x^{2}} \phi(x)+V_{\text {abs }} \phi(x)=E \phi(x)=\frac{(\hbar k)^{2}}{2 m^{\star}} \phi(x), \quad x \in\left[a_{1}, 0\right],
$$

with the boundary conditions $\phi\left(x=a_{1}\right)=0$ and $\phi(x=0)=1$, where we use a simple central finite-difference approximation. Further, let $j_{0}$ be such that $x_{j_{0}}=0$. Then we define the difference $p_{j}=e^{-i \omega \Delta t} u_{j}^{0}-u_{j}^{1}$ for $j=1, \ldots, j_{0}-1$ and $p_{j}=0$ elsewhere, where $u_{j}^{1}$ is the numerical solution of the time-splitting spectral scheme at the grid point $x_{j}$ and 
time $t_{1}=\Delta t$. The factor $e^{-i \omega \Delta t}$ corrects the phase shift after one time step. The solution at time $t_{n}$ is then computed from

$$
U_{j}^{n}=u_{j}^{n}+e^{-i(n-1) \omega \Delta t} p_{j}, \quad j=0, \ldots, M-1, n \geq 1 .
$$

This process is illustrated in Figure 3 for $E=16.74 \mathrm{meV}, \delta=40 \mathrm{~nm}, \triangle x=0.25 \mathrm{~nm}$, and a time step size of $\triangle t=0.25 \mathrm{fs}$. The real part of the incoming plane wave pulse is shown for various times. We see that the pulse spreads from the left to the right lead and equals a plane wave in the physical domain $[0,80] \mathrm{nm}$. In Figure 3 we also show the complete computational domain which is much larger than the physical interval.
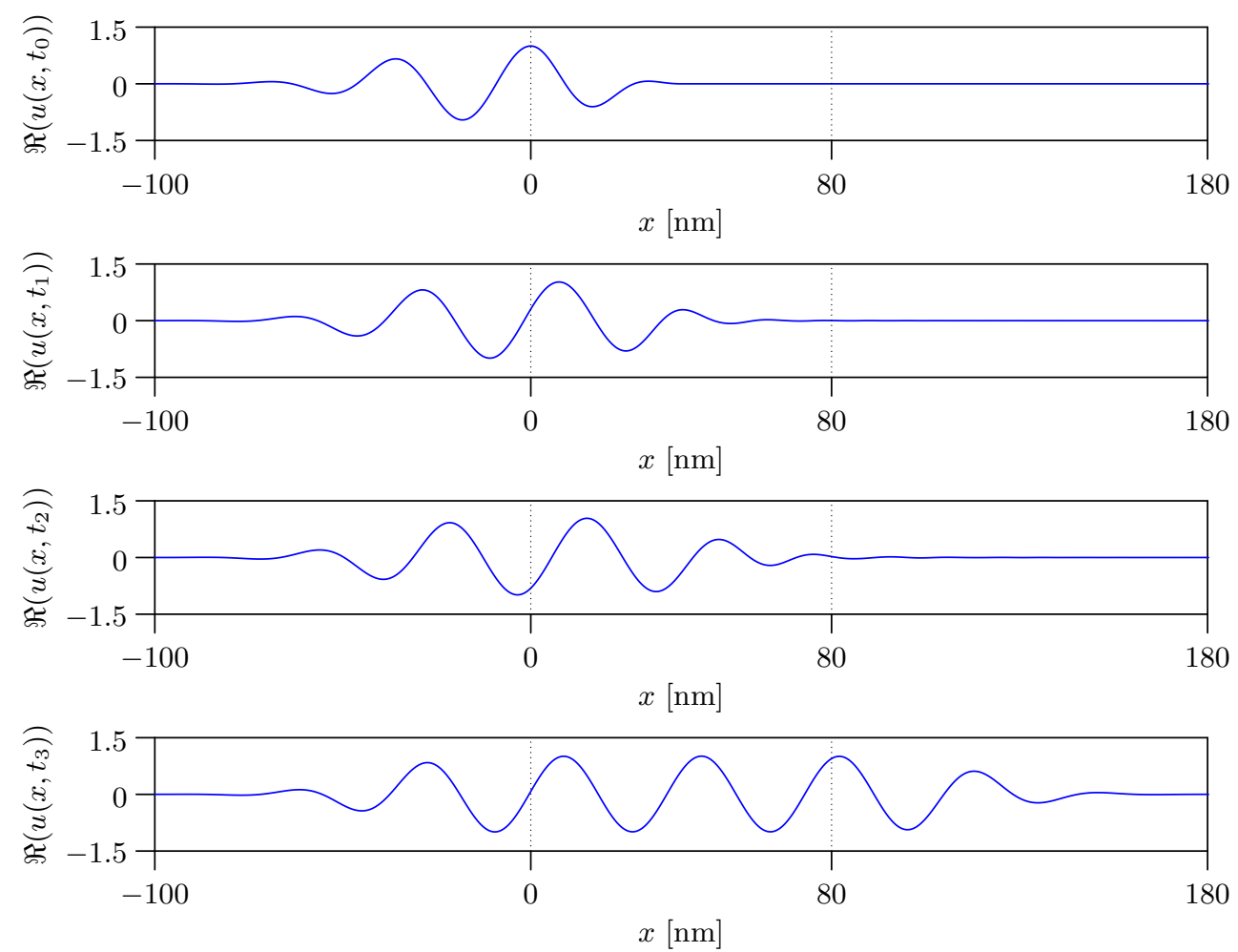

Figure 3: Real part of the incoming plane wave pulse at times $0 \mathrm{fs}, 50 \mathrm{fs}, 100 \mathrm{fs}$, and $5 \mathrm{ps}$.

In several space dimensions, we assume that the incoming pulse travels in the $x_{1^{-}}$ direction, whereas the electrons are confined in the other directions. Then the incoming plane wave pulse is the product of the one-dimensional pulse, as described above, in the $x_{1}$-direction and the solution to the stationary Schrödinger equation in the $x_{2}$-direction (and $x_{3}$-direction if $d=3$ ). 


\section{Numerical simulations}

\subsection{Numerical test in one space dimension}

As a test of the numerical scheme described in Section 2, we consider the evolution of the incoming plane wave pulse in the interval $[0,80 \mathrm{~nm}]$ with a Gaussian external potential

$$
V(x)=V^{\star} e^{-0.02\left(\frac{x-x_{0}}{\mathrm{~nm}}\right)^{2}}, \quad x \in[0,80 \mathrm{~nm}],
$$

placed at $x_{0}=40 \mathrm{~nm}$ (see Figure 4 ). The maximum value of the potential $V^{\star}$ equals the energy of the incoming plane wave $E=16.74 \mathrm{meV}$. The dotted line in Figure 4 represents the scattering state computed with the quantum transmitting boundary method $[20,30]$ on a very fine grid with $\triangle x=0.0025 \mathrm{~nm}$. This is taken as a reference solution for the expected scattering state. The solid line is the numerical solution of the time-splitting spectral method using $\triangle x=0.25 \mathrm{~nm}$ and $\triangle t=0.25 \mathrm{fs}$. Apparently, the solution converges to the scattering state computed with the quantum transmitting boundary method. The relative error in the $\ell^{2}$ norm between the particle densities at various times is shown in Figure 5.
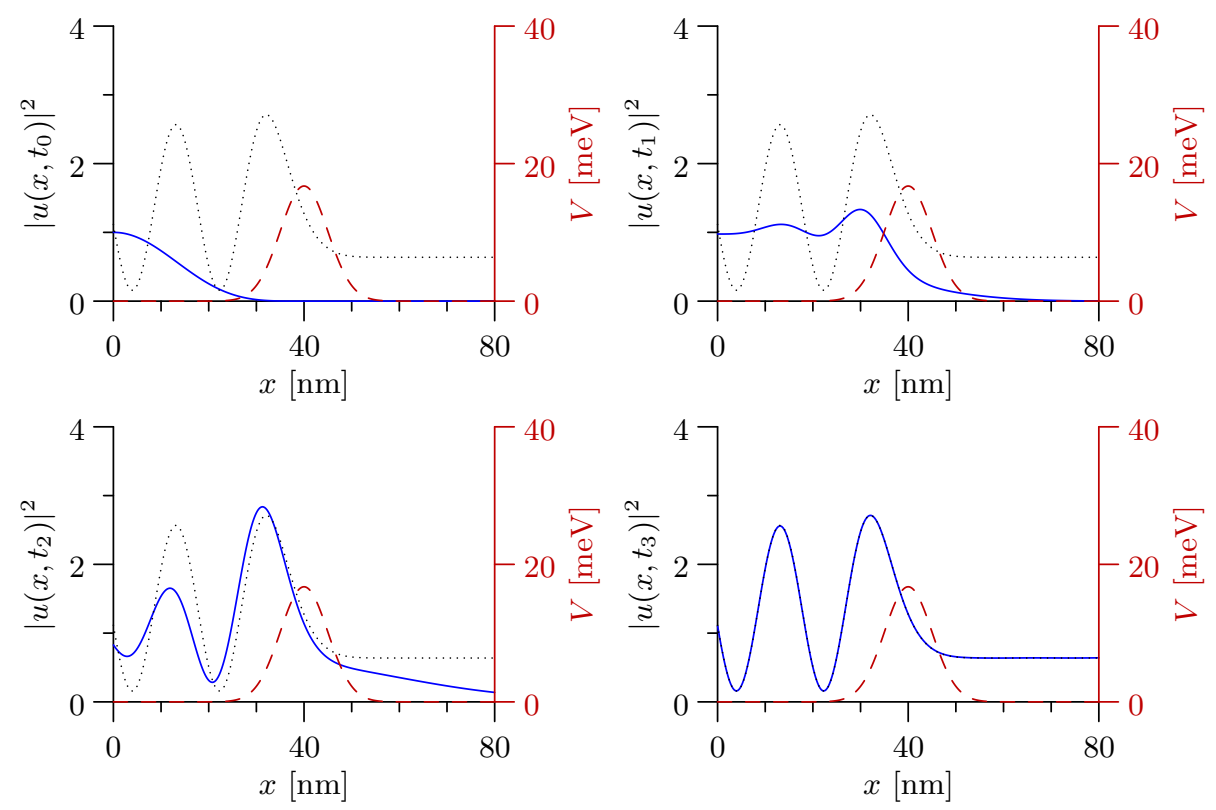

Figure 4: Evolution of the incoming plane wave pulse in the presence of a Gaussian external potential (dashed line) at times $0 \mathrm{fs}, 100 \mathrm{fs}$ (upper row) and $200 \mathrm{fs}, 5 \mathrm{ps}$ (lower row). Solid line: numerical solution computed with the spectral method. Dotted line: scattering state computed with the quantum transmitting boundary method.

Next, we present the numerical convergence rates with respect to the time and space discretization parameters. The relative error is here computed from the difference of the numerical solution and a reference solution in the $\ell^{2}$ norm. 


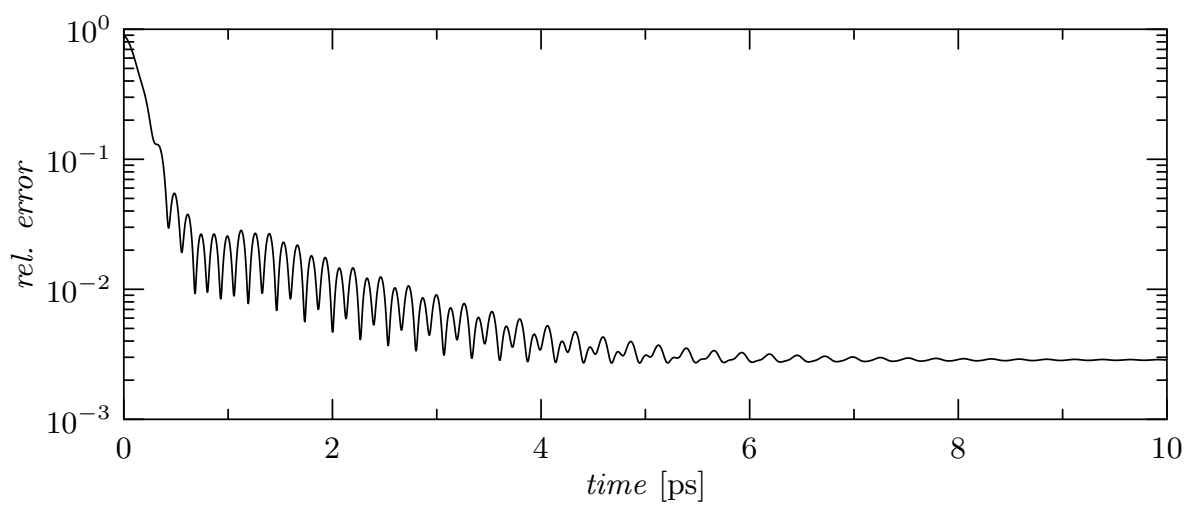

Figure 5: Relative error between the spectral approximation and the solution of the quantum transmitting boundary method in the $\ell^{2}$ norm at various times.

The reference solution is obtained from a Crank-Nicolson finite-difference scheme in combination with discrete transparent boundary conditions (TBC); see Section 1. More precisely, a homogeneous TBC is used at $x=80 \mathrm{~nm}$, whereas an inhomogeneous TBC is used at $x=0 \mathrm{~nm}$, modeling the incoming plane wave pulse. For further details, we refer to [4]. The numerical scheme is unconditionally stable and completely reflection-free at the boundary [6]. Taking $\triangle x=0.0025 \mathrm{~nm}$ and $\Delta t=0.005$ as discretization parameters, we calculate a very accurate reference solution at $t=500 \mathrm{fs}$ which corresponds to 100,000 time steps. As in each time step a convolution of size $n$ (time step number) has to be computed, this calculation is very expensive.

Now we perform the same simulation as above until $t=500 \mathrm{fs}$ with the time-splitting spectral method, where we use different time step sizes $\Delta t$ but a fixed spatial discretization $\triangle x=0.025 \mathrm{~nm}$. The relative error in the $\ell^{2}$ norm between the numerical solution and the reference solution is shown in Figure 6 (left). Here we distinguish between the Trotter time splitting (triangles) and the Strang time splitting (squares). Obviously, the Strang splitting is superior to the Trotter splitting. When the time step size is getting very small, both numerical schemes yield approximately the same error. This is probably due to the influence of the complex absorbing potential outside of the device domain. The convergence rate of the Trotter splitting for moderate time step sizes is approximately 1.1, which corresponds to the first-order time splitting. The convergence rate of the Strang splitting for moderate time step sizes is approximately 2.0, which corresponds to the secondorder time splitting. Next, we repeat the experiment with variable spatial discretizations $\triangle x$ and a fixed time step size $\triangle t=0.1 \mathrm{fs}$. The relative errors are presented in Figure 6 (right).

We notice that the convergence rate obtained in [8] for the Trotter time-splitting spectral method without absorbing potentials reads as follows [8, Thm. 4.1]:

$$
\left\|u\left(t_{n}\right)-u_{I}^{n}\right\|_{L^{2}} \leq C_{m} \frac{T}{\triangle t}\left(\frac{1}{M}\right)^{m}+C T \triangle t \quad \text { for all } m \in \mathbb{N}, t_{n} \leq T
$$

where $u$ is the solution to the Schrödinger equation (1) with periodic boundary conditions 
and $u_{I}^{n}$ is the time-splitting spectral approximation, obtained from

$$
u_{I}^{n}(x)=\frac{1}{M} \sum_{k=-M / 2}^{M / 2-1} \widehat{u}_{k}^{n} e^{i \mu_{k}\left(x-a_{1}\right)} .
$$

The constant $C_{m}>0$ depends on the $L^{\infty}$ norm of the $m$-th derivative of $V$ and the $L^{2}$ norm of the $m$-th derivative of $u$, and $C>0$ contains the operator-splitting error. It is difficult to compare our numerical convergence rate with the theoretical result, since we would need to include the error due to the absorbing potential and since the value of $C_{m}$ is not easy to compute. However, on a graphical level, the solution obtained from the timesplitting spectral method cannot be distinguished from the reference solution as illustrated in Figure 7.
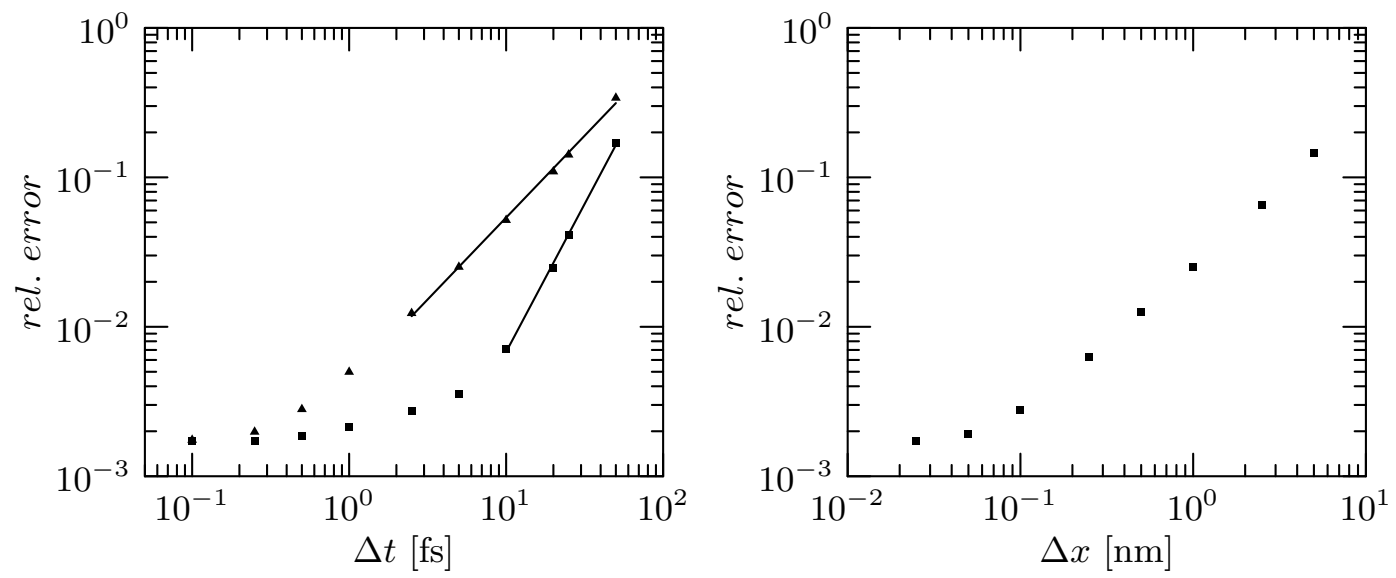

Figure 6: Relative errors for various time step sizes $\Delta t$ (left) and spatial discretizations $\triangle x$ (right) at time $\mathrm{t}=500 \mathrm{fs}$. Triangles: Trotter time-splitting approximation, squares: Strang time-splitting approximation. 

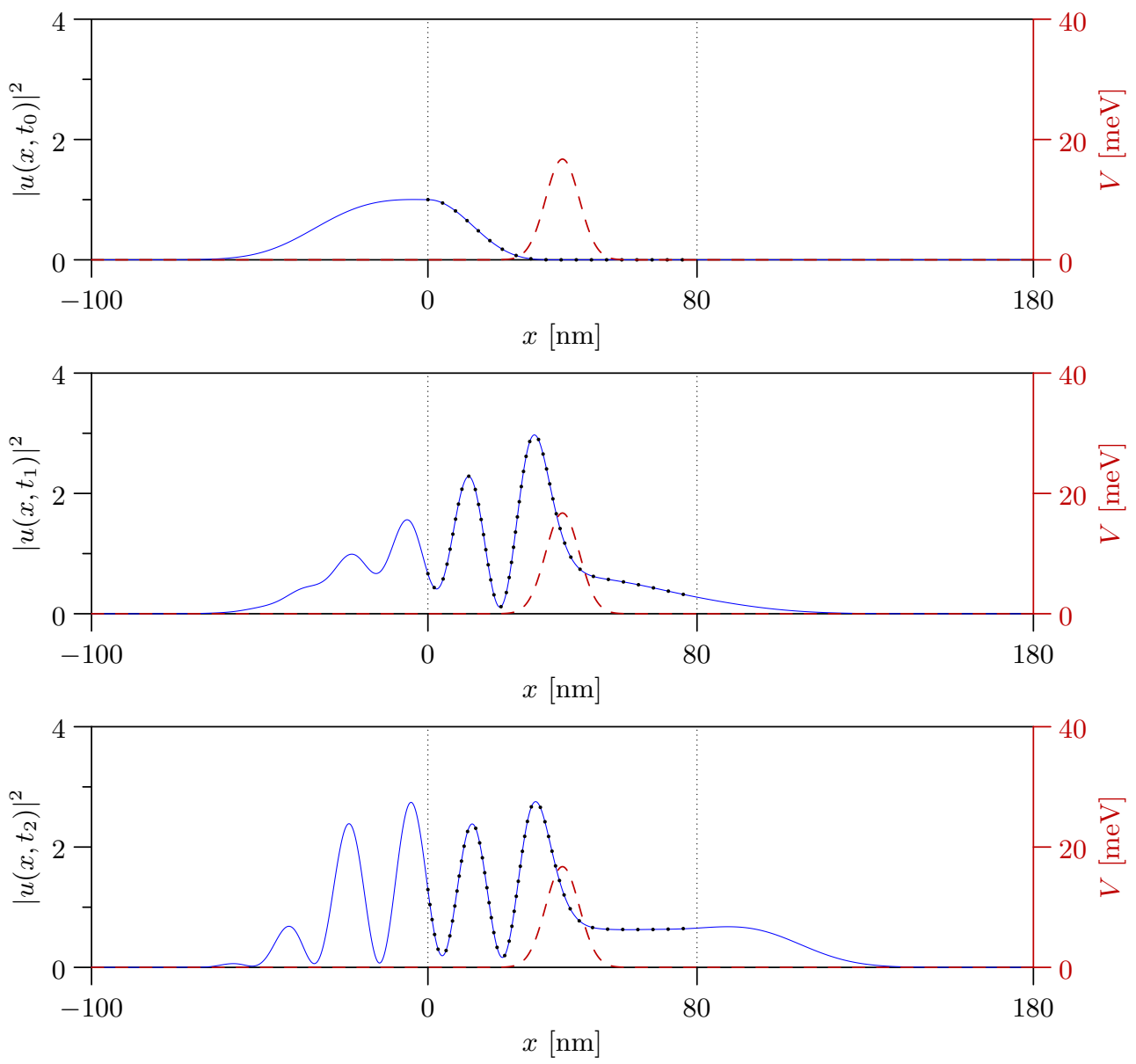

Figure 7: Evolution of a reference solution and the solution computed with the spectral method at times $0 \mathrm{fs}, 250 \mathrm{fs}$ and $500 \mathrm{fs}$. The reference solution is obtained from a CrankNicolson discretization in combination with discrete transparent boundary conditions. The discretization parameters are $\triangle x=0.0025 \mathrm{~nm}$ and $\triangle t=0.005 \mathrm{fs}$. The time-splitting spectral approximation is computed with $\triangle x=0.25 \mathrm{~nm}$ and $\triangle t=0.25 \mathrm{fs}$. Solid line: time-splitting spectral method, dotted line: reference solution.

\subsection{Simulation of two-dimensional quantum waveguides}

The electron transport in a quantum T-stub waveguide is based on the interference of the particles. The electrons have two main paths, one straight path from source to drain and another path going by the stub (Figure 8). The gate voltage modifies the penetration of the electron wave function in the lateral stub. The trajectories interfere constructively or deconstructively, depending on the stub length [2]. Thus, there are two states: the transmission is (almost) one, corresponding to an on-state, or zero, corresponding to an off-state. In this sense, the device acts as a nanoscale switch, also referred to as a quantum (interference) transistor [2]. The electron gas is assumed to be confined in two space dimensions realized by $\mathrm{Al}_{0.3} \mathrm{Ga}_{0.7} \mathrm{As} / \mathrm{GaAs}$ heterostructures such that it is sufficient to solve 
the two-dimensional Schrödinger equation (see the next subsection for three-dimensional simulations).

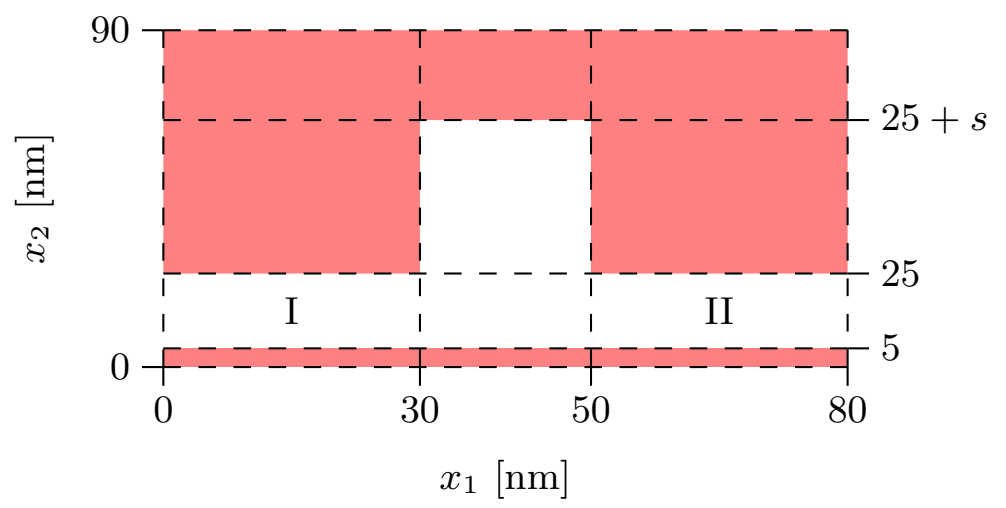

Figure 8: Geometry of the quantum stub transistor.

The geometry of the simulated quantum transistor is similar to that of $[7,10]$, see Figure 8. The length of the transistor is $80 \mathrm{~nm}$, the width of the stub is $20 \mathrm{~nm}$ and its length varies between $20 \mathrm{~nm}$ and $60 \mathrm{~nm}$. The computational domain is the rectangle $\Omega=[-100,180] \times[0,90] \mathrm{nm}^{2}$, and the periodic boundary conditions are imposed on that domain. In contrast to the simulations in $[7,10]$, the heterostructure is not modeled by hard walls and square corners but by a smooth, finite confining potential with maximal value $1 \mathrm{eV}$. The profile of the potential is depicted in Figure 9. The material is GaAs with the effective mass $m^{*}=0.067 m_{0}$.

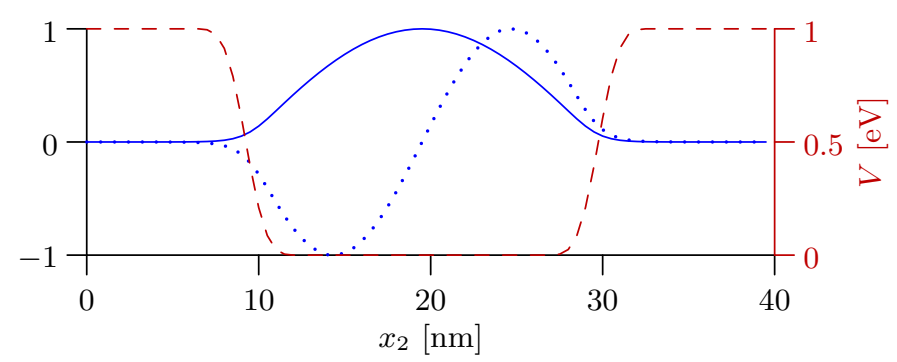

Figure 9: Profile of the confining potential (dashed line), the ground state with $E_{0}=$ $13.16 \mathrm{meV}$ (solid line) and the first excited state with $E_{1}=52.33 \mathrm{meV}$ (dotted line).

Due to the translation invariance of the waveguide problem without stub in the $x_{1}$ direction, the stationary full wave function can be separated as

$$
u_{k, n}\left(x_{1}, x_{2}\right)=e^{i k x_{1}} \phi_{n}\left(x_{2}\right),
$$

where the exponential part corresponds to a plane wave associated to the wave vector $k$. The energy is given by the sum of the energy corresponding to $\phi_{n}$ and the wave energy in $x_{1}$-direction, $E=E_{n}+(\hbar k)^{2} / 2 m^{*}$. The wave function $\phi_{n}$ is a solution to the stationary 
Schrödinger equation

$$
-\frac{\hbar^{2}}{2 m^{*}} \frac{\partial^{2} \phi_{n}}{\partial x_{2}^{2}}+V\left(x_{2}\right) \phi_{n}=E_{n} \phi_{n}
$$

with periodic boundary conditions. This equation is solved by a simple central finitedifference scheme together with the sparse eigenvalue solver eigs of Matlab. The ground state $\phi_{0}$ and the first excited state $\phi_{1}$ are shown in Figure 9. The wave functions decrease exponentially at the transition to the finite potential, whereas in the case of hard walls, they vanish at the walls. The ground state has the energy $E_{0}=13.16 \mathrm{meV}$, which is not much smaller than the energy of the ground state $E_{0}^{*}=14.03 \mathrm{meV}$ corresponding to the infinite square well case.

The electrons are continuously fed into the lead using the plane wave pulse described in Section 2.3. We notice that the angular frequency $\omega=E / \hbar$ occuring in the factor $e^{-i \omega \Delta t}$ is computed by employing the total energy $E$. The incoming wave has the energy $E=29.9 \mathrm{meV}$ (as in [7]), comprised of the ground-state energy $E_{0}=13.16 \mathrm{meV}$ to confine the electrons in the channel and the kinetic energy $16.74 \mathrm{meV}$. This means that we perform the simulation using the lowest transversal mode only. We have chosen $\triangle x_{1}=\triangle x_{2}=$ $0.5 \mathrm{~nm}$ corresponding to $M_{1}=560$ and $M_{2}=160$ grid points. The time step size equals $\triangle t=0.25$ fs.

In this subsection, we are interested in the stationary behavior in order to compare our simulation results with those of $[7,10]$. A (quasi) steady state is reached after about $2 \mathrm{ps}$ within 67 seconds computing time on an Intel Core 2 Quad CPU Q9550 with $4 \times 2.8 \mathrm{GHz}$. The corresponding probability densities for stub lengths $s_{1}=22.5 \mathrm{~nm}, s_{2}=31.5 \mathrm{~nm}$, $s_{3}=41 \mathrm{~nm}$, and $s_{4}=50 \mathrm{~nm}$ are shown in Figure 10. The conductance of the transistor with stub lengths $s_{2}$ and $s_{4}$ almost vanishes, i.e., the electrons interfere in such a way that (almost) no electrons can flow through the device. This corresponds to an off-state of the transistor. The transmission becomes maximal at the stub lengths $s_{1}$ and $s_{3}$, corresponding to an on-state of the device.

The transmission through the device depending on the stub length is shown in Figure 11. As above, the incident energy equals $29.9 \mathrm{meV}$. The transmission at time $t_{n}$ is defined as the quotient

$$
\tau^{n}=\frac{\sum_{j=0}^{M_{2}-1}\left|u_{\ell, j}^{n}\right|^{2}}{\sum_{j=0}^{M_{2}-1}\left|u_{r, j}^{0}\right|^{2}},
$$

where $u_{k, j}^{n}$ approximates the wave function $u\left(a_{1}+k \triangle x_{1}, j \triangle x_{2}, t_{n}\right)$ and $\ell$ and $r$ are the indices of the grid points at $x_{1}=0$ and $x_{1}=80 \mathrm{~nm}$. Since the numerical solution does not reach a perfect steady state, the transmission is averaged over the time interval from $5 \mathrm{ps}$ to $6 \mathrm{ps}$. Interestingly, we find the off- and on-states $(31.5 \mathrm{~nm}$ and $41.0 \mathrm{~nm}$, respectively) at almost the same stub lengths as in [10] (32 nm and $40.5 \mathrm{~nm})$, although we employ a smoothed confining potential in contrast to the hard-wall potential of [10]. This shows that the solution using a smoothed potential does not differ significantly from the hardwall solution.

As a second example we simulate a single-branch coupler, which is an example of a multiport structure. The coupler consists of four ports, coupled through a small window in 

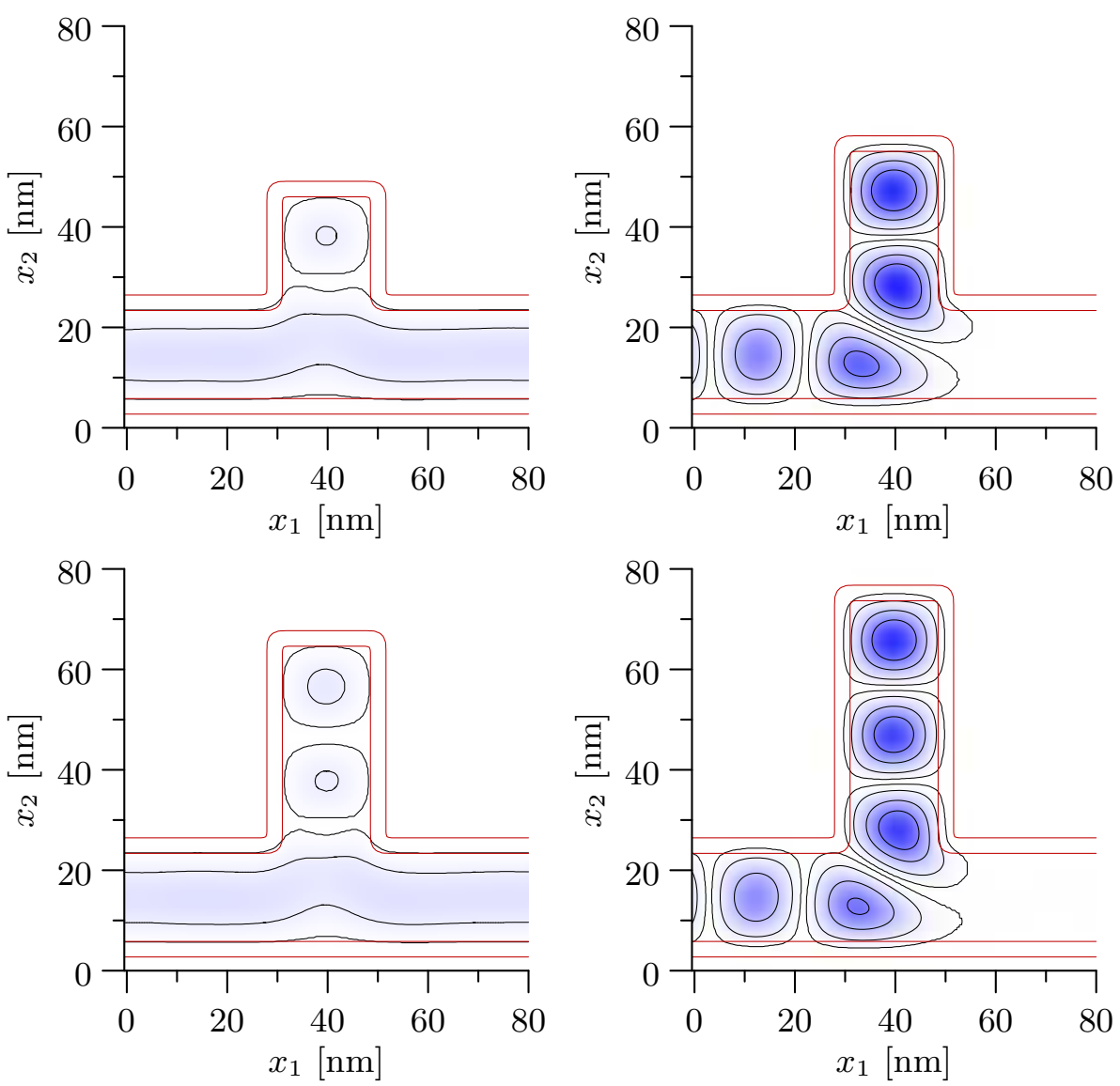

Figure 10: Probability densities in the quantum transistor for stub lengths $22.5 \mathrm{~nm}, 31.5 \mathrm{~nm}$ (upper row) and $41 \mathrm{~nm}, 50 \mathrm{~nm}$ (lower row) at $t=2 \mathrm{ps}$. Isolines of the density $|u|^{2}$ are shown at the values $0.05,0.5,2,4$, and those of the potential $V$ at $0.05 \mathrm{eV}, 0.95 \mathrm{eV}$.

the middle; see Figure 12. A detailed study of the conductance in a single-branch coupler with hard walls can be found in [10]. The geometry is taken from [10]. The window width equals $w=30 \mathrm{~nm}$, and the computational domain is $[-100,180] \times[0,100] \mathrm{nm}^{2}$. We employ as in the previous example a smoothed potential with the maximal value $1 \mathrm{eV}$.

The mono-energetic plane wave pulse is injected at the lower left lead of the device (port I). The other ports are numbered counter-clockwise. Depending on the incident energy (and the window width), we obtain different steady states and different transmissions $\tau_{12}$, $\tau_{13}$, and $\tau_{14}$, which are defined similarly as in (5). The transmissions as a function of the kinetic energy are shown in Figure 13. The transmission $\tau_{14}$ from port I to port IV is maximal at $16.5 \mathrm{meV}$, i.e., the injected electrons leave most likely the device at port IV. The corresponding probability density is illustrated in Figure 14 (left). In Figure 14 (right), the density for a window width $w=51 \mathrm{~nm}$ is shown. Compared to the results of [10], we find the same interference patterns, and $\tau_{14}$ reaches its maximum value for almost the same energy (which equals $16.37 \mathrm{meV}=30.4 \mathrm{meV}-14.03 \mathrm{meV}$ in [10, p. 710]), although 


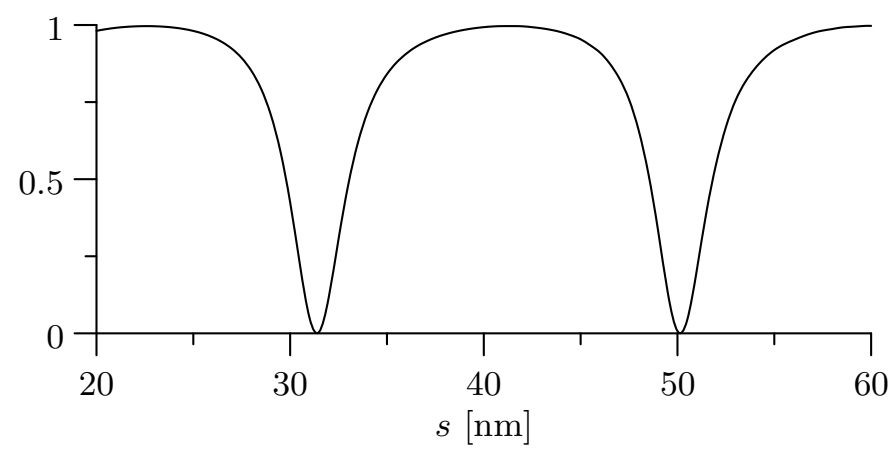

Figure 11: Transmissions of the quantum transistor, averaged over the time interval from 5 ps to $6 \mathrm{ps}$, depending on the stub length $s$.

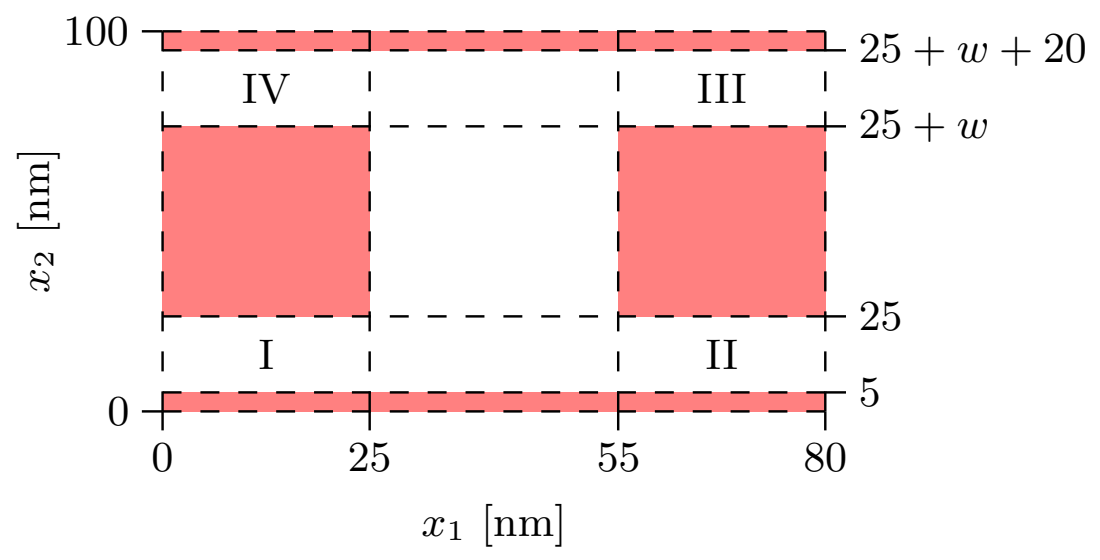

Figure 12: Geometry of the single-branch coupler.

we employ a smoothed potential.

We notice that all simulations are performed with the same absorbing potential (4) with $V_{0}=50 \mathrm{meV}$. This choice turned out to be appropriate if the kinetic energy of the electrons is between $15 \mathrm{meV}$ and $25 \mathrm{meV}$. For different kinetic energies, the absorbing potential needs to be adapted, which is clearly a disadvantage of the method compared to, for instance, the method of [7]. However, we stress the fact that, due to the simple absorbing potential technique, our algorithm is very fast, and three-dimensional simulations are feasible (see below).

\subsection{Simulation of a three-dimensional quantum waveguide}

A quantum waveguide has a three-dimensional structure, in which the electrons may be confined in the $\left(x_{1}, x_{2}\right)$-direction. Therefore, we choose the channel height $x_{3}=5 \mathrm{~nm}$, whereas the total length of the quantum transistor is $x_{1}=80 \mathrm{~nm}$ and the waveguide width is $x_{2}=20 \mathrm{~nm}$ (see Figure 15). The stub length is between $33 \mathrm{~nm}$ and $43 \mathrm{~nm}$.

The channel profile is presented in Figure 16. The ground state and the first excited state are computed from the two-dimensional Schrödinger equation. The three-dimensional 


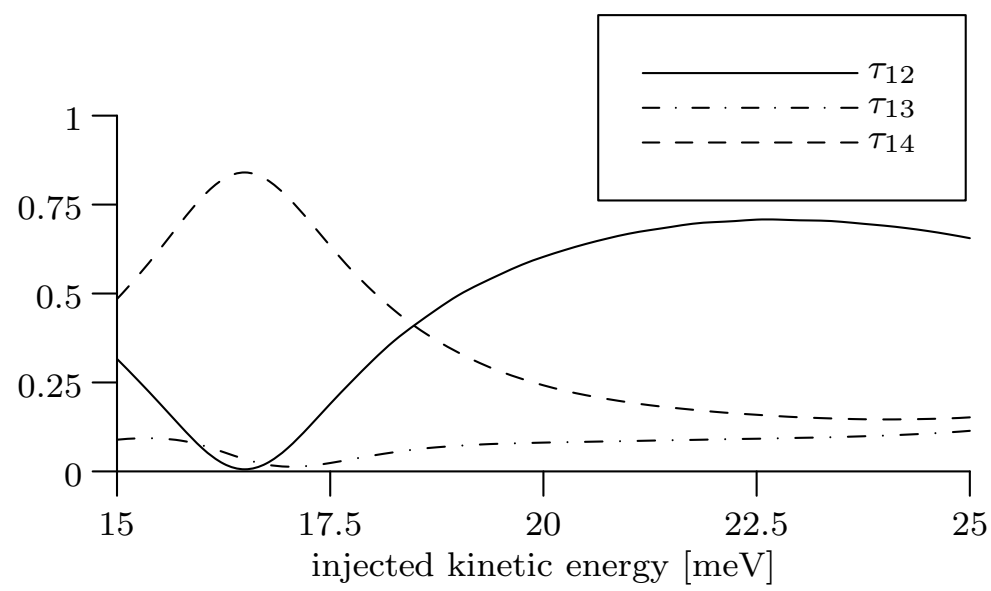

Figure 13: Transmissions from port I to ports II, III, and IV, respectively, of the singlebranch coupler, averaged over the time interval from 5 ps to $6 \mathrm{ps}$.

simulations are performed on a grid with $\triangle x_{1}=\triangle x_{2}=\triangle x_{3}=0.5 \mathrm{~nm}$ and with time step size $\Delta t=0.25 \mathrm{fs}$. Complex arrays of size $560 \times 150 \times 30$ are needed to store the numerical wave function, the potential, and three auxiliary variables, using only $322 \mathrm{MB}$ memory capacity. The simulation presented below took about four hours computing time on an Intel Core 2 Quad CPU Q9550 with $4 \times 2.8 \mathrm{GHz}$.

Figures 17-18 show the transient behavior of the probability densities in the quantum transistor at different times. At time $t=0$, the stub length is fixed at $s_{1}=33 \mathrm{~nm}$. It takes about 2 ps to reach the (quasi) steady state. The stub length is chosen such that the device is in the off-state. From 2 ps to $2.5 \mathrm{ps}$, the sub length is continuously increased to $s_{2}=43 \mathrm{~nm}$. After another transient phase, the solution converges to a new (quasi) steady state. The electrons flow through the device which is in the on-state. The stub length is decreased from $5.5 \mathrm{ps}$ to $6 \mathrm{ps}$ to $s_{1}$, and the simulation is stopped at $7.5 \mathrm{ps}$.

Figures 17-18 are realized as follows. Every 25 time steps, the isosurfaces of the electron density are calculated using the Matlab built-in function isosurface. This yields vertices and faces of a triangle mesh. The normals of the isosurface vertices are derived from the Matlab command isonormals. The matrices describing the faces, vertices, and normals are needed to create a mesh representation which can be included in the POV-Ray language [28]. In order to visualize the inner and outer surfaces of the electron density at 0.05 and 0.25 , the outer surface is made almost transparent. In total, 1200 scene description files have been rendered. The rendered images are used to create a movie illustrating the switching process, see http://www . jungel at.vu, item "Simulations". The rendering process took about six hours computing time on the above mentioned CPU.

\section{Bibliography}

[1] X. Antoine, A. Arnold, C. Besse, M. Ehrhardt, and A. Schädle. A review of transparent and artificial boundary conditions techniques for linear and nonlinear Schrödinger 

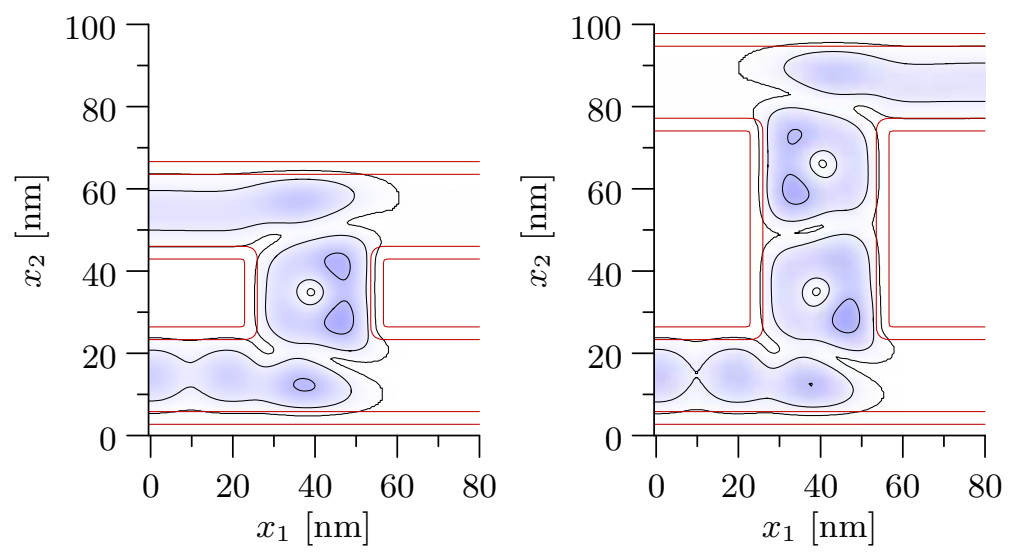

Figure 14: Probability densities of the single-branch coupler at time $t=2 \mathrm{ps}$. The kinetic energy of the electrons injected in the lower left part is $16.5 \mathrm{meV}$. The window width equals $w=20 \mathrm{~nm}$ (left) and $w=51 \mathrm{~nm}$ (right).

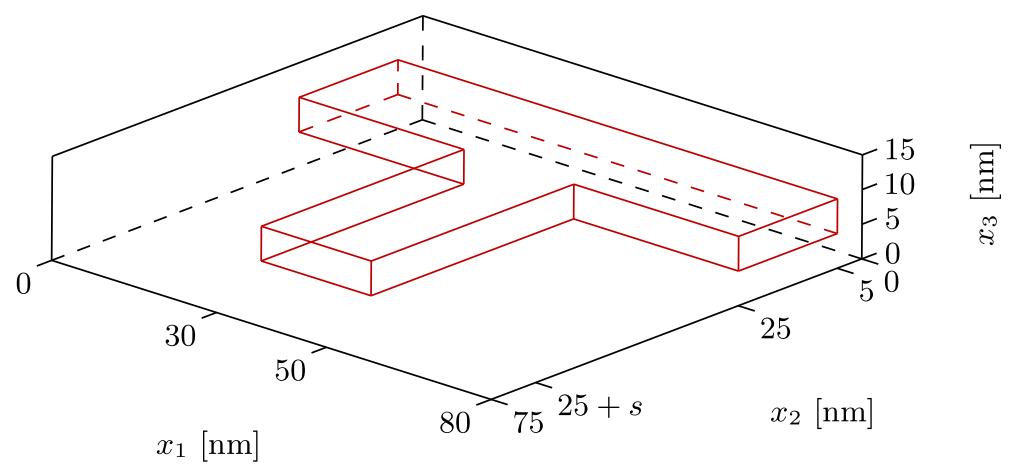

Figure 15: Geometry of the quantum transistor.

equations. Commun. Comput. Phys. 4 (2008), 729-796.

[2] J. Appenzeller, C. Schroer, T. Schäpers, A. von der Hart, A. Förster, B. Lengeler, and H. Lüth. Electron interference in a T-shaped quantum transistor based on Schottkygate technology. Phys. Rev. B 53 (1996), 9959-9963.

[3] A. Arnold. Numerically absorbing boundary conditions for quantum evolution equations. VLSI Design 6 (1998), 313-319.

[4] A. Arnold. Mathematical concepts of open quantum boundary conditions. Transp. Theory Stat. Phys. 30 (2001), 561-584.

[5] A. Arnold, M. Ehrhardt, and M. Schulte. Numerical simulation of quantum waveguides. Preprint, Vienna University of Technology, Vienna, Austria, 2009.

[6] A. Arnold, M. Ehrhardt, and I. Sofronov. Discrete transparent boundary conditions 

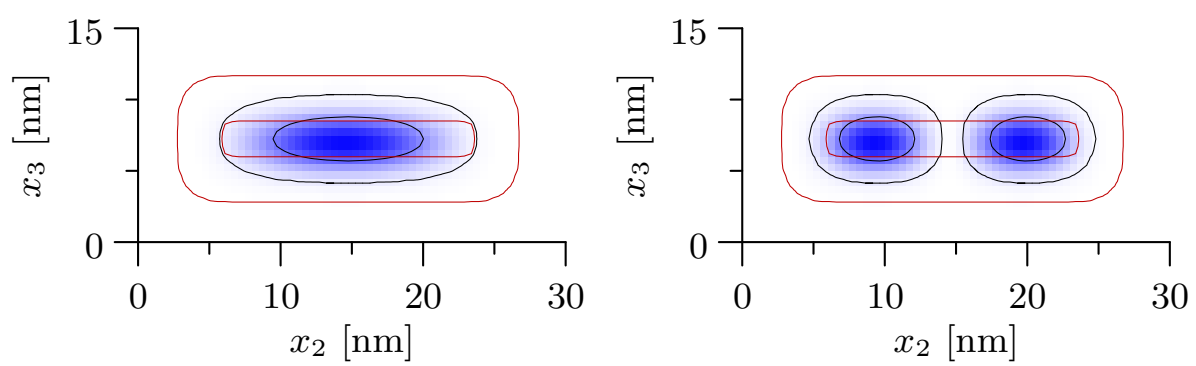

Figure 16: Cross section of the three-dimensional quantum transistor, isolines of the potential at $0.05 \mathrm{eV}$ and $0.95 \mathrm{eV}$, and the densities of the ground state with energy $E_{0}=176.98 \mathrm{meV}$ and the first excited state with $E_{1}=215.79 \mathrm{meV}$, together with the isolines at 0.05 and 0.5 .

for the Schrödinger equation: Fast calculation, approximation, and stability. Commun. Math. Sci. 1 (2003), 501-556.

[7] A. Arnold and M. Schulte. Transparent boundary conditions for quantum-waveguide simulations. Math. Comput. Simul. 79 (2008), 898-905.

[8] W. Bao, S. Jin, and P. Markowich. On time-splitting spectral approximations for the Schrödinger equation in the semiclassical regime. J. Comput. Phys. 175 (2002), 487-524.

[9] J. Berenger. A perfectly matched layer for the absorption of electromagnetic waves. J. Comput. Phys. 114 (1994), 185-200.

[10] L. Burgnies, O. Vanbésien, and D. Lippens. An analysis of wave patterns in multiport quantum waveguide structures. J. Phys. D: Appl. Phys. 32 (1999), 706-712.

[11] C. Cheng, J.-H. Lee, K.-H. Lim, H. Massoud, and Q.-H. Liu. 3D quantum transport solver based on perfectly matched layer and spectral element methods for the simulation of semiconductor nanodevices. J. Comput. Phys. 227 (2007), 455-471.

[12] M. Ehrhardt and A. Arnold. Discrete transparent boundary conditions for the Schrödinger equation. Riv. Mat. Univ. Parma. 6 (2001), 57-108.

[13] M. Frigo and S. Johnson. The design and implementation of FFTW3. Proc. IEEE 93 (2005), 216-231.

[14] J.-Y. Ge and J. Zhang. Use of negative complex potential as absorbing potential. J. Chem Phys. 108 (1998), 1429-1433.

[15] H. Han, D. Yin, and Z. Huang. Numerical solutions of Schrödinger equations in $\mathbb{R}^{3}$. Numer. Meth. Part. Diff. Eqs. 23 (2007), 511-533. 

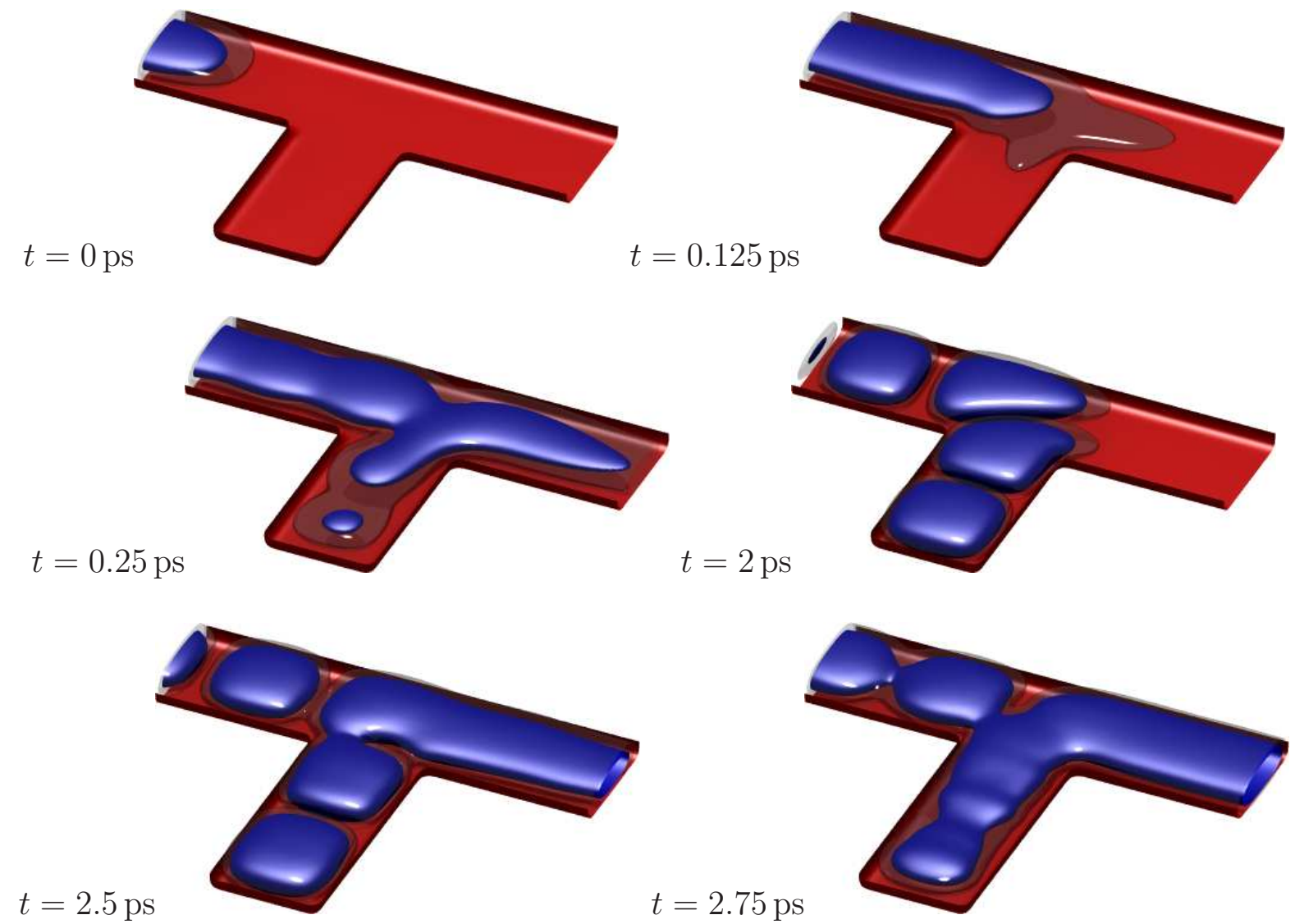

Figure 17: Probability densities in the three-dimensional quantum transistor at various times. The lower half of the isosurface at $0.5 \mathrm{eV}$ of the potential and two isosurfaces at 0.05 and 0.25 of the electron density are shown.

[16] A. Hussain and G. Roberts. Procedure for absorbing time-dependent wave functions at low kinetic energies and large bandwidths. Phys. Rev. A 63 (2000), 012703 (11 pages).

[17] T. Jahnke and C. Lubich. Error bounds for exponential operator splittings. BIT 40 (2000), 735-744.

[18] A. Jüngel. Transport Equations for Semiconductors. Lect. Notes Phys. 773. Springer, Berlin, 2009.

[19] C. Leforestier and R. Wyatt. Optical potential for laser induced dissociation. J. Chem. Phys. 78 (1983), 2334-2344.

[20] C. Lent and D. Kirkner. The quantum transmitting boundary method. J. Appl. Phys. 67 (1990), 6353-6359.

[21] C. Lubich. On the multistep time discretization of linear initial-boundary value problems and their boundary integral equations. Numer. Math. 67 (1994), 365-389. 

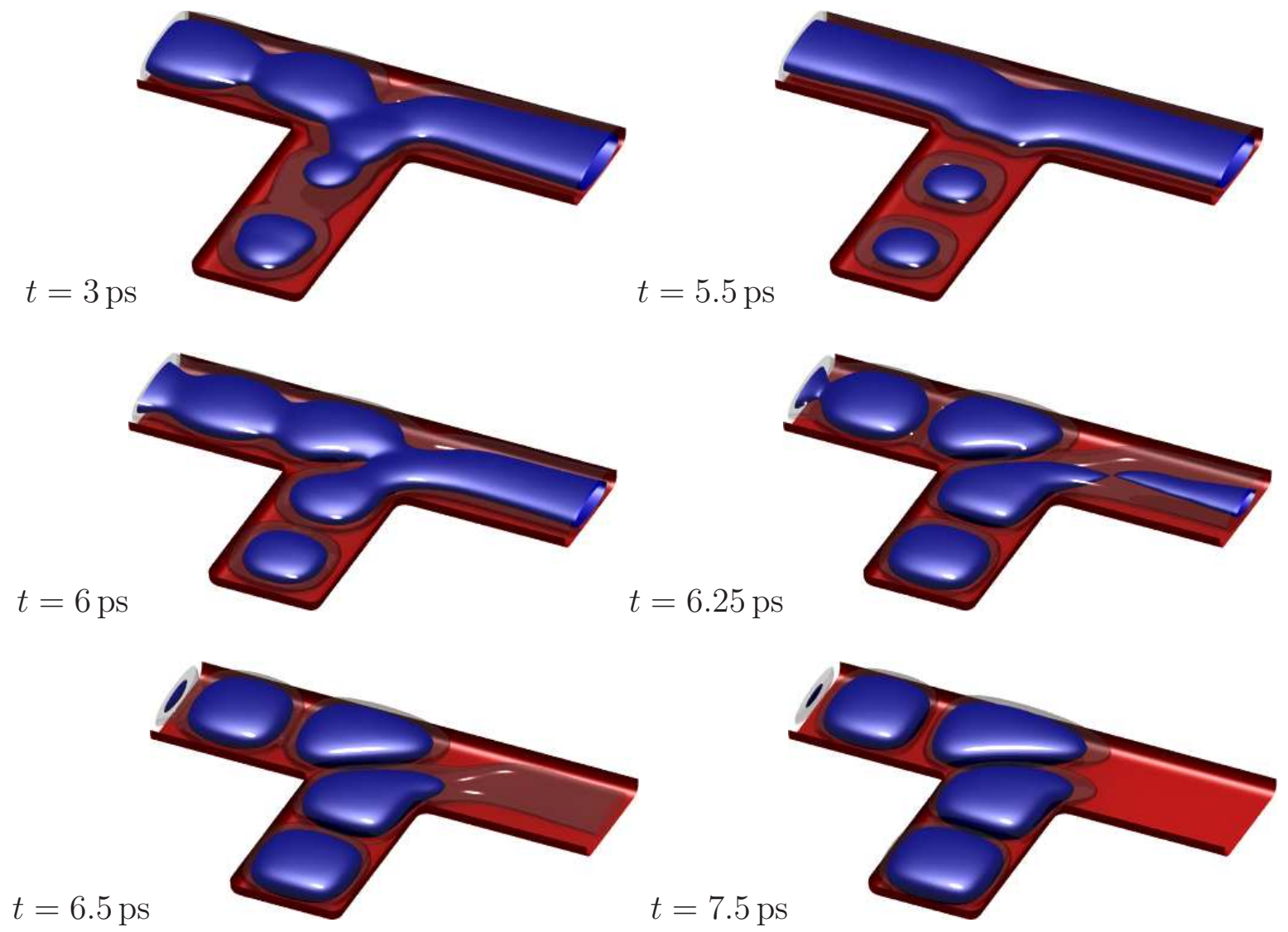

Figure 18: Probability densities in the three-dimensional quantum transistor at various times. The lower half of the isosurface at $0.5 \mathrm{eV}$ of the potential and two isosurfaces at 0.05 and 0.25 of the electron density are shown.

[22] C. Lubich and A. Schädle. Fast convolution for nonreflecting boundary conditions. SIAM J. Sci. Comput. 24 (2002), 161-182.

[23] S. Mahapatra and N. Sathyamurthy. Negative imaginary potentials in time-dependent molecular scattering. J. Chem. Soc., Faraday Trans. 93 (1997), 773-779.

[24] M. Nedjalkov, D. Vasileska, E. Atanassov, and V. Palankovski. Ultrafast Wigner transport in quantum wires. J. Comput. Electron. 6 (2007), 235-238.

[25] D. Neuhauser and M. Baer. The time-dependent Schrödinger equation: Application of absorbing boundary conditions. J. Chem. Phys. 90 (1989), 4351-4355.

[26] A. Nissen and G. Kreiss. An optimized perfectly matched layer for the Schrödinger equation. Preprint, Uppsala Universitet, Sweden, 2009.

[27] D. Pathria and J. Morris. Pseudo-spectral solution of nonlinear Schrödinger equation. J. Comput. Phys. 87 (1990), 108-125. 
[28] Persistence of Vision Raytracer (Version 3.6), http://www . povray . org.

[29] E. Polizzi and N. Ben Abdallah. Self-consistent three-dimensional models for quantum ballistic transport in open systems. Phys. Rev. B 66 (2002), 245301 (9 pages).

[30] E. Polizzi and N. Ben Abdallah. Subband decomposition approach for the simulation of quantum electron transport in nanostructures. J. Comput. Phys. 202 (2005), 150180 .

[31] A. Schädle. Numerische Behandlung transparenter Randbedingungen für die Schrödinger-Gleichung. Master Thesis, Universität Tübingen, Germany, 1998.

[32] F. Schmidt and P. Deuflhard. Discrete transparent boundary conditions for the numerical solution of Fresnels equation. Comput. Math. Appl. 29 (1995), 53-76.

[33] M. Shin. Three-dimensional quantum simulations of multigate nanowire field effect transistors. Math. Comput. Simul. 79 (2008), 1060-1070.

[34] A. Soffer and C. Stucchio. Open boundaries for the nonlinear Schrödinger equation. J. Comput. Phys. 225 (2007), 1218-1232.

[35] F. Sol, M. Macucci, U. Ravaioli, and K. Hess. Theory for a quantum modulated transistor. J. Appl. Phys. 66 (2989), 3892-3906.

[36] A. Svizhenko, M. Anantram, T. Govindan, B. Biegel, and R. Venugopal. Twodimensional quantum mechanical modeling of nanotransistors. J. Appl. Phys. 91 (2002), 2343-2354.

[37] A. Thean, and J.-P. Leburton. Stark effect and single-electron charging in silicon nanocrystal quantum dots. J. Appl. Phys. 89 (2001), 2808-2815.

[38] H. Tsuchiya, M. Ogawa, and T. Miyoshi. Wigner function formulation of quantum transport in electron waveguides and its application. Japan. J. Appl. Phys. 30 (1991), 3853-3858.

[39] N. Tsukada, A.D. Wieck, and K. Ploog. Proposal of novel electron wave coupled devices. Appl. Phys. Lett. 56 (1990), 2527-2529.

[40] R. Venugopal, Z. Ran, S. Datta, M. Lundstrom, and D. Jovanovic. Simulating quantum transport in nanoscale transistors: Real versus mode-space approaches. J. Appl. Phys. 92 (2002), 3730-3739.

[41] C. Zheng. A perfectly matched layer approach to the nonlinear Schrödinger wave equation. J. Comput. Phys. 227 (2007), 537-556. 